\title{
A NECESSARY CONDITION THAT TWO FINITE QUASI-FIELDS COORDINATIZE ISOMORPHIC TRANSLATION PLANES
}

\author{
M. L. NARAYANA RAO AND F. W. WILKE
}

Given two quasi-fields it is usually easier to determine whether or not they are isotopic or anti-isotopic than it is to determine whether or not they can coordinatize isomorphic planes. The theorem given here has proved to be quite useful for this purpose. (See, for example, [2].)

A translation plane $\pi$ may always be coordinatized, in the Hall sense [1], by a right quasi-field (Veblen-Weddenburn system). It is known that two quasi-fields which are either isotopic or anti-isotopic coordinatize isomorphic translation planes. This note gives a necessary condition that two finite quasi-fields which are neither isotopic nor anti-isotopic can coordinatize isomorphic translation planes.

For the remainder of this note let $F_{1}, F_{2}$ be finite quasi-fields and $\pi_{1}, \pi_{2}$ the associated translation planes in the sense of Hall [1]. For $i=1,2$ let $F_{i \rho}=\left\{a \in F_{i} \mid a \neq 0\right.$ and $\left.(x y) a=x(y a) \forall x, y \in F_{i}\right\}=$ right nucleus of $F_{i}$ and let $F_{i \mu}=\left\{a \in F_{i} \mid a \neq 0\right.$ and $\left.(x a) y=x(a y)\right\}=$ middle nucleus of $F_{i}$. It is easy to see that for each $a \in F_{i p}$ the mapping $\beta_{a}:(x, y) \rightarrow(x, y a),(m) \rightarrow(m a), Y_{i}=(\infty) \rightarrow Y_{i}$ is a perspectivity of $\pi_{i}$ with center $Y_{i}$ and axis the line $y=0$. The correspondence $a \leftrightarrow \beta_{a}$ is an isomorphism between $F_{i \rho}$ and the set of $Y_{i}-O_{i} X_{i}$ perspectivities of $\pi_{i}$. Also, for each $a \in F_{i \mu}$, the mapping $\gamma_{a}:(x, y) \rightarrow(x a, y),(m)$ $\rightarrow\left(m L_{(a)}^{-1}\right), \quad Y_{i} \rightarrow Y_{i}$ is a $X_{i}-O_{i} Y_{i}$ perspectivity of $\pi_{i}$ and the correspondence $a \leftrightarrow \gamma_{a}$ is an isomorphism between $F_{i \mu}$ and the set of $X_{i}-O_{i} Y_{i}$ perspectivities of $\pi_{i}$.

We will use the conventional notation $Y=(\infty), O=(0,0), X=(0)$. We will also use the symbol $|A|$ to denote the cardinality of a set $A$.

Theorem. Suppose $F_{1}$ and $F_{2}$ are neither isotopic nor anti-isotopic and that $\pi_{1}$ and $\pi_{2}$ are isomorphic. Let $\sigma$ be an isomorphism from $\pi_{1}$ to $\pi_{2}$ with $O_{1} \sigma=O_{2}, X_{1} \sigma=X_{3}, Y_{1} \sigma=Y_{3}$. Let $D=\left\{X_{2}, Y_{2}\right\} \cap\left\{X_{3}, Y_{3}\right\}$. Assume that if there is an isomorphism from $\pi_{1}$ to $\pi_{2}$ such that $D \neq \varnothing$ then $\sigma$ is such an isomorphism. Then

(1) If $|D|=1$ then $\left(\left|F_{1 \rho}\right|,\left|F_{2 \rho}\right|\right)=\left(\left|F_{1 \mu}\right|,\left|F_{2 \mu}\right|\right)=1$.

(2) If $|D|=0$ then $\left(\left|F_{1 \rho}\right|,\left|F_{2 \rho}\right|\right)$ and $\left(\left|F_{1 \mu}\right|,\left|F_{2 \mu}\right|\right) \leqq 2$.

Received by the editors May 12, 1969. 
Proof. Since $F_{1}$ and $F_{2}$ are neither isotopic nor anti-isotopic $|D|<2$. We will prove the theorem for the right nuclei. The proof for the middle nuclei is the same except for the appropriate word replacements. Let $A$ be the set of all $Y_{2}-O_{2} X_{2}$ perspectivities of $\pi_{2}$ and let $B$ be the set of all $Y_{3}-O_{2} X_{3}$ perspectivities of $\pi_{2}$. Then $|A|$ $=\left|F_{2 \rho}\right|$ and $|B|=\left|F_{1 \rho}\right|$. Let $M=\left\{p \in L_{2 \infty} \mid p=X_{2} \alpha\right.$ or $p=Y_{2} \alpha$ for some collineation $\alpha$ of $\left.\pi_{2}\right\}$. Then $A$ is a group of permutations on $M-\left\{X_{2}, Y_{2}\right\}$ such that each orbit contains $\left|F_{2 \rho}\right|$ elements. Thus $|M|=k\left|F_{2 \rho}\right|+2$ for some integer $k$. Also, $B$ is a group of permutations on $M-\left\{X_{3}, Y_{3}\right\}$ with each orbit containing $\left|F_{1 \rho}\right|$ elements so that $|M|=t\left|F_{1 \rho}\right|+|D|$ and so $\left(\left|F_{1 \rho}\right|,\left|F_{2 \rho}\right|\right) \leqq 2$. If $|D|=1$ then $\left(\left|F_{1 \rho}\right|,\left|F_{2 \rho}\right|\right)=1$.

Corollary. In the theorem, if $D=\varnothing$ then the points of $L_{\infty}$ in $\pi_{2}$ or $\left.\pi_{1}\right)$ may be partitioned into three mutually disjoint sets $A_{1}, A_{2}, A_{3}$ such that

$$
\left|\begin{array}{l}
A_{i} \\
A_{i} \\
A_{3}
\end{array}\right| \equiv 0 \bmod \bmod \left|F_{j \rho}\right| \text { for } i, j=1,2 \text {, and } i \neq j, j,
$$

Proof. Since $D=\varnothing,|D|=0$ so that $|M| \equiv 2 \bmod \left|F_{2 \rho}\right|$ and $|M| \equiv 0 \bmod \left|F_{1 \rho}\right|$. Let $M=A_{2}$. Let $A_{1}=\left\{p \in L_{2 \infty} \mid p=X_{3} \alpha\right.$ or $p=Y_{3} \alpha$ for some collineation $\alpha$ of $\left.\pi_{2}\right\}$. Clearly the proof of the theorem with the appropriate change of symbols shows that $\left|A_{1}\right| \equiv 0 \bmod \left|F_{2 \rho}\right|$ and $\left|A_{1}\right| \equiv 2 \bmod \left|F_{1_{\rho}}\right| . D=\varnothing$ implies $A_{1} \cap A_{2}=\varnothing$.

Let $A_{3}=L_{2 \infty}-\left(A_{1} \cup A_{2}\right)$. Then each of $A$ and $B$ acts as a permutation group on $A_{3}$ with orbits of size $\left|F_{2 \rho}\right|$ and $\left|F_{1 \rho}\right|$ respectively. Thus, $\left|A_{3}\right| \equiv 0 \bmod \left(\operatorname{LC} M\left[\left|F_{1 \rho}\right|,\left|F_{2 \rho}\right|\right]\right)$.

\section{REFERENCES}

1. Marshall Hall Jr., The theory of groups, Macmillan, New York, 1959. MR 21 \#1996.

2. M. L. Narayana Rao, D. J. Rodabaugh, J. L. Zemmer and F. W. Wilke, $A$ new class of finite translation planes obtained from the exceptional near-fields, J. Combinatorial Theory (to appear).

University of Missouri-Columbia AND

UNIVERSITY OF MisSOURI-SAINT LOUIS 